\title{
Corrente de Sobrevivência à COVID-19
}

\section{COVID-19 Chain of Survival 2020}

\author{
Sergio Timerman, ${ }^{1}$ Helio Penna Guimarães, ${ }^{2}$ Carlos Eduardo Rochitte, ${ }^{1,3}$ Thatiane Facholi Polastri, ${ }^{1}$ Marcelo \\ Antônio Cartaxo Queiroga Lopes ${ }^{4,5,6}$ \\ Universidade de São Paulo Instituto do Coração, ${ }^{1}$ São Paulo, SP - Brasil \\ Hospital Israelita Albert Einstein, ${ }^{2}$ São Paulo, SP - Brasil \\ Hospital do Coração, ${ }^{3}$ São Paulo, SP - Brasil \\ Hospital Alberto Urquiza Wanderley - Hemodinâmica e Cardiologia Intervencionista, ${ }^{4}$ João Pessoa, PB - Brasil \\ Hospital Metropolitano Dom José Maria Pires, ${ }^{5}$ João Pessoa, PB - Brasil \\ Sociedade Brasileira de Cardiologia, ${ }^{6}$ Rio de Janeiro, RJ - Brasil
}

\section{Sumário}

O termo Corrente de Sobrevivência fornece uma metáfora útil para os elementos do conceito de atendimento às vítimas da COVID 19. Seus cinco elos são:

- CIÊNCIA - Medicina baseada em evidências (políticas públicas e políticas)

- SENSIBILIZAÇÃO - Sensibilização e conscientização da população

- TREINAMENTO - Treinamento de profissionais de saúde individualmente e em equipe

- ESTRUTURA - Equipamento e estruturação do préhospitalar e do hospital no atendimento à COVID-19

- RETOMADA - Retorno do paciente e da equipe

Uma forte Corrente de Sobrevivência pode melhorar as chances de sobrevivência e recuperação para as vítimas da COVID-19.

Realização: Sociedade Brasileira de Cardiologia (SBC), Associação Brasileira de Medicina de Emergência (ABRAMEDE)

Nota: Essa corrente se presta a informar e não a substituir o julgamento clínico do médico que, em última análise, deve determinar o tratamento apropriado para seus pacientes.

\section{Introdução}

Em dezembro de 2019, a Comissão Municipal de Saúde e Saneamento de Wuhan, Província de Hubei, China, relatou um grupo de 27 casos de pneumonia de etiologia desconhecida, sendo 7 graves. Em janeiro de 2020, as autoridades chinesas identificaram um novo vírus da família Coronaviridae, então denominado 'novo coronavírus' ou 2019-nCoV e, posteriormente,

\section{Palavras-chave}

COVID-19; Coronavírus; Betacoronavírus; Medicina Baseada em Evidência; Sobrevivência; Cuidados Médicos.

Correspondência: Sergio Timerman •

Universidade de São Paulo Instituto do Coração - Rua Para, 270 CEP 01243020, São Paulo, SP - Brasil

E-mail: timerman@cardiol.br

Artigo recebido em 04/11/2020, revisado em 04/11/2020, aceito em $04 / 11 / 2020$

DOI: https://doi.org/10.36660/abc.20201171
SARS-CoV-2 (síndrome respiratória aguda grave coronavírus 2). A doença do novo coronavírus foi denominada COVID-19. ${ }^{1}$ Desde então, a COVID-19 alastrou-se, tendo acometido, até a primeira quinzena de outubro, 35.628.628 indivíduos em todo o mundo, sendo os Estados Unidos e o Brasil o epicentro, com 9.385.506 e 5.566.049 casos, respectivamente. No mesmo período, foram registrados 1.215.756 óbitos e o Brasil permanecia em segundo lugar nesse ranking, com 160.496 mortes. $^{2}$

As medidas de emergência para tratar pacientes com COVID-19 e conter o surto são a principal prioridade em cada um dos países. No entanto, é provável que essas medidas resultem em danos colaterais para pacientes com outras doenças agudas, além de agravar as condições econômicas e sociais da população.

A Corrente de Sobrevivência à COVID-19 refere-se à cadeia de eventos que deve ocorrer em rápida sucessão para maximizar as chances de sobrevivência à doença e de restabelecimento de fluxos de saúde e sociais. Trata-se de uma metáfora simples para demonstrar ao público seu papel vital no controle da COVID-19, bem como o papel dos profissionais da saúde nessa corrente. Sugere que cada elo seja crítico e interdependente e que a corrente de sobrevivência seja forte quando consolidada em todos os seus elos, cuja abordagem efetiva possa ajudar a salvar vidas.

\section{Princípios gerais}

Aprendendo com o drama mundial da COVID-19, este artigo aborda os principais aspectos do papel dos técnicos de saúde que, em paralelo com o corpo gerencial e médico, têm respondido às necessidades em constante mudança, proporcionando ambientes de cuidado adequado para os infectados e proteção para outros pacientes e operadores.

Esta explosão pandêmica, de fato, destacou a importância dos sistemas de saúde e hospitais em primeiro lugar e sua gestão constante como parte relevante de toda a governança neste momento dramático e complexo.

Este artigo tem por objetivo criar e descrever detalhadamente os componentes estruturais de uma Corrente de Sobrevivência à COVID-19. As recomendações contidas neste documento são baseadas nas evidências disponíveis no momento da sua elaboração e na opinião de especialistas. O conhecimento em relação à COVID-19 evolui de forma dinâmica e rápida; logo, os protocolos para reintrodução com segurança de atendimentos médicos e de procedimentos invasivos e não invasivos estão em constante evolução e adaptação. Este projeto foi idealizado pela Sociedade Brasileira de Cardiologia e pela Associação Brasileira 


\section{Carta Científica}

de Medicina de Emergência, como uma fonte de referência para seus associados. As recomendações apresentadas, contudo, não devem ser usadas como única base para a definição de protocolos locais, devendo outras fontes atualizadas ser consideradas à medida que o conhecimento na área evolui.

O mesmo esforço sistemático, organizado e coordenado em uma comunidade continua sendo a recomendação mais forte que podemos fazer para salvar mais pessoas acometidas pela COVID-19. A metáfora dos elos de uma corrente provou ser bemsucedida em muitos aspectos na ressuscitação cardiopulmonar., A utilização de uma Corrente de Sobrevivência à COVID-19 pode identificar pontos fracos nos 'elos do sistema de atenção e combate à pandemia e assim auxiliar as comunidades a otimizarem o tratamento de pacientes críticos acometidos pela COVID-19.

Nesse contexto, todos passam a ser fundamentais e devem trabalhar em harmonia. Principais gestores de saúde, equipes de profissionais de saúde, do serviço técnico, da engenharia clínica, da gestão de risco, da farmácia, entre outros, constituem um sistema, cujo trabalho, à semelhança daquele de uma orquestra, requer gerenciamento dinâmico e operação em campo.

A sobrevivência à forma grave da COVID-19 depende de uma sequência de intervenções críticas. Se uma dessas ações críticas for negligenciada ou atrasada, diminuem as chances de sobrevivência. Usamos o termo Corrente de Sobrevivência para descrever essa sequência. A Corrente de Sobrevivência à COVID-19 possui cinco elos interdependentes, como mostrado na Figura 1.

- CIÊNCIA: Medicina baseada em evidências (políticas e políticas públicas) - A cadeia de comando no sistema de saúde e o desafio COVID-19

Um ponto essencial é ocupar espaço no debate público, aproveitando o que a pandemia nos ensina como ocasião para uma avaliação objetiva sobre a governança dos sistemas de saúde. A discussão necessariamente continuará, como deve ser em uma democracia, mas é fundamental que as lições do período dramático sejam aprendidas.
A medicina baseada em evidências consiste no uso explícito e consciencioso das melhores evidências científicas para tomada de decisão no cuidado ao paciente. A medicina baseada em evidências busca unir a experiência do médico, os valores e desejos dos pacientes e a melhor evidência científica disponível. ${ }^{5-7}$ A pandemia da COVID-19 tem promovido sensíveis repercussões pessoais e políticas no âmbito da sociedade, impactando discussões calorosas e verdadeiros embates no agonismo e antagonismo de novos/velhos fármacos ou tratamentos. Esses cenários, no entanto, jamais modificam a demanda por melhores evidências de ensaios clínicos randomizados especificamente desenhados para determinar as modalidades de tratamento baseadas em evidências para reduzir a propagação dessa doença e prevenir o fardo de surtos futuros. ${ }^{5-7}$

\section{- SENSIBILIZAÇÃO: Sensibilizar e conscientizar a população}

Embora muitas terapias tenham sido sugeridas, até o momento nenhuma opção específica é capaz de tratar a COVID-19 ou agir de forma profilática quanto à infecção por SARS-CoV-2. A única intervenção atualmente viável e comprovada para reduzir a taxa de contágio parece ser o uso de medidas estritas de isolamento social para a população em geral. ${ }^{5}$ Os resultados de revisões sistemáticas e meta-análises apoiam o distanciamento físico de pelo menos 1 metro e fornecem estimativas quantitativas para modelos de rastreamento de contatos. O uso ideal de máscaras faciais, a higienização adequada das mãos e a proteção de contato em face/olhos nos ambientes públicos parecem promover impacto. ${ }^{5,6}$ Maclntyre et al., ${ }^{8}$ em estudo randomizado comparando o uso de máscaras de tecido com máscaras cirúrgicas em profissionais de saúde, verificaram um número significativamente maior de acometimento respiratório entre os usuários de máscaras de tecido. Estudos e recomendações posterOiores, ${ }^{8-12}$ envolvendo simulação aplicada à distribuição populacional e transmissão da COVID-19, demonstraram que o uso contínuo de máscara facial (filtrante de 20-50\% do ar exalado) pela população, mesmo assintomática, reduz significativamente a disseminação da COVID-19, com efeitos benéficos independentemente de potenciais grupos

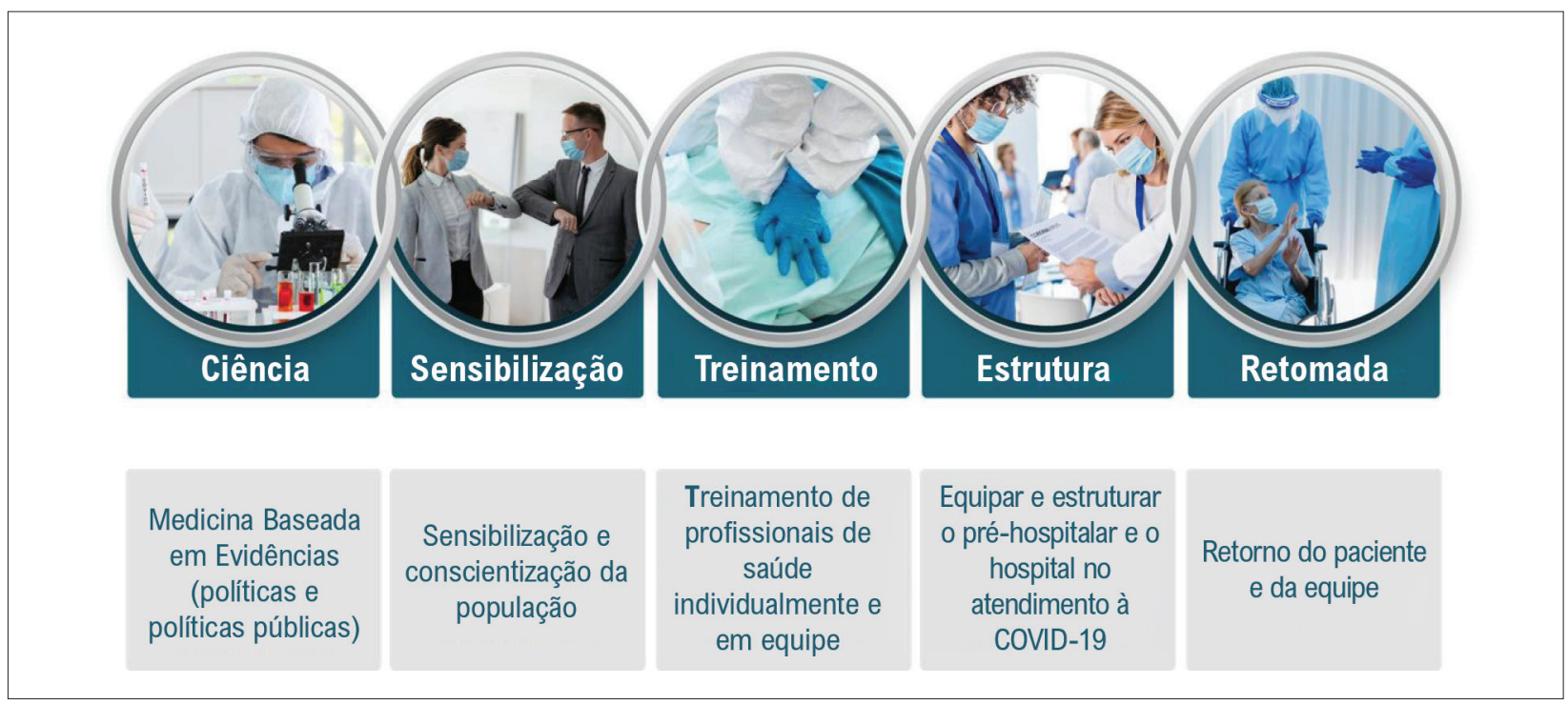

Figura 1 - Corrente de Sobrevivência à COVID-19. 
da população associados a maior risco. Tal redução pode ser potencialmente otimizada quando combinada ao distanciamento social. Portanto, é plausível e necessário considerar que todos os indivíduos utilizem máscaras quando expostos a aglomerações e outras situações de alto risco, em especial devido à maior transmissibilidade no período precoce assintomático da doença.

- TREINAMENTO: Treinamento dos profissionais de saúde (individual e em equipe)

A interrupção da educação médica permanente estendeuse muito além de residência médica. O cancelamento de congressos, cursos e simpósios, a redução de equipes clínicas pelo afastamento de profissionais contaminados, a relutância quanto ao deslocamento para treinamentos, os confinamentos, o intenso acúmulo de funções e o aumento da carga de trabalho na pandemia da COVID-19 exigiram a implementação de tecnologias, assim como a adaptação e a ação imediata para minimizar a lacuna educacional. ${ }^{13,14}$

As teleconferências já haviam sido introduzidas como meio útil de educação continuada muito antes da COVID-19. No entanto, com a pandemia, o uso proeminente dessa tecnologia envolveu estratégias de ensino entre instituições, ${ }^{13-18}$ tornando-se o meio fundamental para a continuidade da educação clínica permanente e provando sua utilidade. Aplicativos diversos e multimídias de reunião virtual permitiram que departamentos clínicos implementassem palestras e sessões clínicas, bem como faculdades de medicina continuassem suas atividades, além de permitir que hospitais/diretorias clínicas continuassem a realizar seus relatórios e discussões de casos.

O ensino médico baseado em simulação manteve-se como estratégia adequada durante a pandemia, com adequações tais quais a redução do número de participantes, implementação de equipamentos de proteção individual e desinfecção de manequins. ${ }^{19-22}$

- ESTRUTURA: Equipamento e estruturação do préhospitalar e do hospital no atendimento à COVID-19

Diante da necessidade imediata de lidar com uma doença nova, a telemedicina propiciou aprendizado interdisciplinar e assistência a distância. ${ }^{23}$ Por exemplo, no Brasil, médicos intensivistas e emergencistas puderam atuar em consultorias para o atendimento de pacientes com COVID-19, contribuindo indiretamente para o manejo clínico baseado em evidências. ${ }^{24-26}$ O projeto Tele UTI, executado de forma colaborativa por cinco hospitais privados filantrópicos para atendimento de 2.500 leitos de unidade de terapia intensiva do Sistema Único de Saúde liderado pelo Hospital Israelita Albert Einstein, consiste em visita médica diária aos pacientes internados nas unidades de terapia intensiva, com ênfase nos casos de síndrome respiratória aguda grave e suspeita de COVID-19. ${ }^{27}$

\section{- RETOMADA: Retorno do paciente e da equipe}

O momento da reintrodução dos atendimentos médicos rotineiros e da vida em sociedade deve estar alinhado a políticas sociais e seguir as recomendações das autoridades competentes. É essencial que a comunidade médica e a sociedade permaneçam vigilantes e atentas a novas evidências e possíveis novos surtos. ${ }^{26}$

As seguintes medidas são indispensáveis: adequação da estrutura física com o objetivo de garantir distanciamento físico, através de demarcações sinalizadas no chão associadas à utilização de barreiras físicas no ambiente, como painéis de acrílico ou vidro; disponibilização do álcool em gel; sinalizações, como cartazes, placas e pôsteres, em locais estratégicos com informações sobre higienização das mãos, etiqueta da tosse e principais sinais e sintomas da COVID-19; ${ }^{26-29}$ e, fundamentalmente, o retorno da economia e a adequação social ao 'novo hoje'.

Rumo ao pós-COVID-19: Lições e desafios para hospitais e infraestruturas de saúde

Há evidências de possíveis repetições de ataques virais em um futuro próximo. A prevenção e a preparação são essenciais, especialmente para o setor da saúde.

\section{Conclusão}

A primeira lição é que este dramático período que vivemos exige coragem para mudar: quem trabalha na área da saúde tem que repensar os modelos arquitetônicos. Os técnicos fizeram milagres ao adaptar os hospitais atuais para atender drasticamente às novas necessidades e, no futuro, devem ser incluídos nos processos de planejamento e design. A governança dos sistemas de saúde tem que considerar a necessidade de menos fragmentação, de forte coordenação nacional. Há que se levar em conta que neste momento temos que caminhar de forma sustentável, tendo como metas a prevenção e a preparação, além de um desenvolvimento econômico focado no respeito às pessoas, à comunidade e ao meio ambiente. Assim, devemos nos lembrar da fala de Maquiavel e aproveitar uma crise dramática.

O conceito da Corrente de Sobrevivência à COVID-19 ressalta vários princípios importantes e, se qualquer elo da cadeia for inadequado, as taxas de sobrevivência serão baixas. A fraqueza nos componentes do sistema é a principal explicação para a variabilidade nas taxas de sobrevivência.

Embora todos os elos devam ser fortes, sempre surge a pergunta inevitável: qual o mais importante? Certamente o reconhecimento da emergência e o início da cadeia são essenciais e, se ninguém reconhecer a emergência e começar a agir, a sobrevivência será baixa.

Como a 'Estrutura' é a única intervenção 'suficiente', ou seja, é o elo que trata a COVID-19, é frequentemente proclamado como 'o fator mais importante na determinação da sobrevida'. A eficácia não pode ser identificada examinando um elo individual, pois todo o sistema deve ser avaliado. A verdade, no entanto, é ainda mais satisfatória e mais condizente com o conceito de Corrente de Sobrevivência, em que cada elo é importante.

Uma forte Corrente de Sobrevivência pode melhorar as chances de sobrevivência e recuperação das vítimas de COVID-19.

\section{Contribuição dos autores}

Concepção e desenho da pesquisa: Timerman S; Redação do manuscrito: Timerman S, Guimarães HP, Rochitte CE, Polastri TF; Revisão crítica do manuscrito quanto ao conteúdo intelectual importante: Timerman S, Guimarães HP, Rochitte CE, Polastri TF, Lopes MACQ.

\section{Potencial conflito de interesses}

Declaro não haver conflito de interesses pertinentes. 


\section{Carta Científica}

\section{Fontes de financiamento}

O presente estudo não teve fontes de financiamento externas.

\section{Referências}

1. Jimenez AJ, Garcia DE. Recomendaciones de Actuación frente a casos de infección por El nuevo coronavirus (SARS-COV-2). In: Guimarães HP, Lobo SMA, Shubert DU, Dal-Pizzol F. Recomendações em Medicina de Emergência e Medicina Intensiva para o atendimento a COVID-19. São Paulo: Editora dos Editores,2020, p.269-320.

2. COVID-19 Dashboard by the Center for Systems Science and Engineering (CSSE) at Johns Hopkins University (JHU).[Acesso em 04 ezembro 2020] Disponivel em : https://coronavirus.jhu.edu/map.html

3. Gullo A. Cardiac Cardiac arrest, chain of survival and Utstein style Eur J Anaesthesiol. 2002 Sep; 19(9): 624-33.

4. Iwami T, Nichol G, Hiraide A, Hayashi Y, Nishiuchi T, Kajino K,et al. Continuous improvements in "chain of survival" increased survival after outof-hospital cardiac arrests: a large-scale population-based study. Circulation. 2009 Feb 10;119(5):728-34

5. Remuzzi A, Remuzzi G. COVID-19 and Italy: what next? Lancet 2020; 395: 1225-8.

6. Pascarella G, Strumia A, Piliego C, Bruno F, Del Buono R. COVID-19 diagnosis and management: a comprehensive review. J Intern Med. 2020 May 13: 10.1111.

7. Malay SD. COVID-19, Pandemic, and Social Distancing J Foot Ankle Surg. 2020 May-June; 59(3): 447-8.

8. Maclntyre CR, Seale H, Dung TC, Tham DC, Hlen ND. A cluster randomised trial of cloth masks compared with medical masks in healthcare workers. BMJ Open, 2015;5(4):e006577.

9. Chu DK, AkI AE, Duda S, Solo K, Yaacoub S, Schünemann HJ. COVID-19 Systematic Urgent Review Group Effort (SURGE) study authorsPhysical distancing, face masks, and eye protection to prevent person-to-person transmission of SARS-CoV-2 and COVID-19: a systematic review and metaanalysis. Lancet. 2020 Jun 27;395(10242):1973-87

10. Eikenberry SE, Mancuso M, Iboi E, Phan T, Einkenberry K, Kuang Y, e al.et al. To mask or not to mask: Modeling the potential for face mask use by the general public to curtail the Covid-19 pandemic. Infect Dis Model, 2020;5:293-308.

11. Mahase E. Covid-19: What is the evidence for cloth masks?. BMJ, 2020;369:m1422.

12. Javid B, Weekes MP, Matheson NJ. Covid-19: should the public wear face masks?. BMJ, 2020;369:m1442.

13. Dedeilia A , Sotiropoulos MG, HanrahanJG, Deep J, Dedeilias P, Sideris M. Medical and Surgical Education Challenges and Innovations in the COVID-19 Era: A Systematic Review. In Vivo. 2020 Jun;34(3 Suppl):1603-11.

14. Tomlinson J, Shaw T, Munro A, Johnson R, Madden DL, Phillips R, et al. How does tele-learning compare with other forms of education delivery? A systematic review of tele-learning educational outcomes for health professionals. N S W Public Health Bull .2013;24:70-5.

15. Spitzer RF, Kives S, Ornstein M, Caccia N, Stephens D, Flood C, et al. Videoconferencing for resident teaching of subspecialty topics: the pediatric

\section{Vinculação acadêmica}

Não há vinculação deste estudo a programas de pósgraduação.

and adolescent gynecology experience at the Hospital for Sick Children. J Pediatr Adolesc Gynecol .2008;21(6):343-6.

16. Stokes DC: Senior medical students in the COVID-19 response: An opportunity to be proactive. Acad Emerg Med.2020; 27(4):343-5.

17. Rakowsky S, Flashner BM, Doolin J, Reese Z, Shpilsky J, Yang S, et al. Five Questions for residency leadership in the time of COVID-19: Reflections of chief medical residents from an internal medicine program. Acad Med. 2020;95(8):1152-4.

18. Kogan M, Klein SE, Hannon CP, Nolte MT: Orthopaedic education during the COVID-19 pandemic. J Am Acad Orthop Surg.2020;323(21):2131-2.

19. Rose S: Medical Student Education in the Time of COVID-19. JAMA, 2020 323(21):2131-2.

20. Mian A, Khan S: Medical education during pandemics: a UK perspective. BMC Med 18(1):100.

21. Brandão CFS, Vaccarezza G, Bizario JCS, Goes AFT. Clinical simulation strategies for knowledge integration relating to initial critical recognition and management of COVID-19 for use within continuing education and health related academia in Brazil: a descriptive study. Sao Paulo Med. J., 138(5):385-92.

22. Deng A, Wang JJ, Tsui BCH. Keeping trainees safe in a pandemic: the evolving role of medical simulation training. Can J Anaesth. 2020;67(9):1292-3.

23. Hollander JE, Carr BG. Virtually Perfect? Telemedicine for Covid-19. N Engl J Med. 2020;382(18):1679-81.

24. Guimarães HP, Couto TB, Corrêa TD, Rodrigues RR, Timerman S, Garrido $\mathrm{AP}$, et al. Cardiorespiratory arrest care in critically patients with suspected or confirmed infection by COVID-19: recommendations and training. Einstein (São Paulo);2020 agosto 2020 (in press).

25. Carvalho CRR, Scudeller PG, Rabello G, Gutierrez MA, Jatene FB. Use of telemedicine to combat the COVID-19 pandemic in Brazil. Clinics. 2020;75:e2217

26. Hospitais Proadi-SUS. Tele UTI.[Acesso em 202016 agosto] Disponível em:https://hospitais.proadi-sus.org.br/covid19/conheca-as-iniciativas-daforca-tarefa/12/tele-uti

27. Lockhart SL, Naidu JJ, Badh CS, Duggan LV. Simulation as a tool for assessing and evolving your current personal protective equipment: lessons learned during the coronavirus disease (COVID-19) pandemic. Can J Anesth. 2020; 67(7):895-6.

28. Bittencourt MS, Generoso G, Melo PHMC, Bittencourt DP, Miranda EJFP, Mesquita ET, et al. Protocolo de reconexão dos serviços de cardiologia com os pacientes durante a pandemia de COVID-19: Posicionamento-Protocolo de reconexão dos serviços de cardiologia com os pacientes durante a pandemia de COVID-19-2020. [Acesso em 202003 Outubro). Disponível em: https://www. portal.cardiol.br/post/protocolo-de-reconex\%C3\%A3o-dos-servi\%C3\%A7osdecardiologia-com-os-pacientes-durante-a-pandemia-de-covid-19.

29. Timerman S, Starbelli TMV, Facciolli T, Kalil RK. Atlantica Hotels Safe \& Clean Protocol e Chancela InCor .[ Accessed in 2020 Oct 20] Available from:https://www.atlanticahotels.com.br/comunicado-covid-19/ 